\title{
Bilateral absence of the transverse sinuses with fenestrated superior sagittal sinus draining through enlarged occipital and marginal sinuses
}

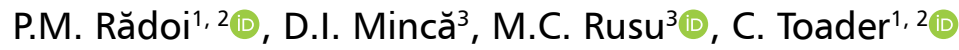 \\ ${ }^{1}$ Faculty of General Medicine, "Carol Davila" University of Medicine and Pharmacy, Bucharest, Romania \\ ${ }^{2}$ National Institute of Neurology and Neurovascular Diseases, Bucharest, Romania \\ ${ }^{3}$ Division of Anatomy, Faculty of Dental Medicine, "Carol Davila" University of Medicine and Pharmacy, Bucharest, Romania
}

[Received: 8 June 2021; Accepted: 7 July 2021; Early publication date: 21 July 2021]

\begin{abstract}
The endothelial-lined dural venous sinuses collect blood from the brain, meninges, and calvaria and drain it to the internal jugular veins. The adult drainage pathway of the venous sinuses confluent is commonly via the transverse and sigmoid sinuses to the jugular bulb. The occipital (OS) and marginal (MS) sinuses are well-represented before birth, in most cases. During a retrospective study of the computed tomography angiograms of a 64-year-old female was found a rare combination of variants of the posterior fossa sinuses. The confluence of the dural venous sinuses was rhomboidal and drained superiorly the superior sagittal sinus, and inferiorly a well-represented OS. The transverse sinuses were aplastic, on the right side, and hypoplastic on the opposite side. The OS further drained into the MS which, on each side, emptied into the respective jugular bulb. On each side a condylar vein left the junction of the sigmoid sinus and jugular bulb. Such posterior fossa drainage, exclusively on the OS-MS pathway, should be kept in mind when transections of the venous sinuses are intended during neurosurgical approaches of the foramen magnum. The OS-MS drainage is rather a persisting foetal pattern. The bilateral anatomical exclusion of the transverse sinuses is an added condition to spare the OS and MS. (Folia Morphol 2022; 81, 3: 781-784)
\end{abstract}

Key words: computed tomography, dura mater, venous sinus, foramen magnum, posterior cranial fossa

\section{INTRODUCTION}

The endothelial-lined dural venous sinuses collect blood from the brain, meninges, and calvaria and drain it to the internal jugular veins (IJVs) [2]. The superior group of the dural venous sinuses is the main one and consists of the sagittal sinuses, superior (SSS) and inferior, the straight sinus (StS), the occipital sinus (OS), the transverse sinus (TS) and the sigmoid sinus (SiS) [2]. The TS and the SiS represent the lateral sinus. The confluence of sinus- es (torcular Herophili) lies in front of the internal occipital protuberance.

The OS lies in the attached margin of the falx cerebelli and drains into the confluence of sinuses $[2,3]$. Its presence was variably detected in $18-81.7 \%$ of cases, depending on the methods of study [6]. On computed tomography angiograms the OS was short, rudimenta$r y$, and untraceable to the foramen magnum in 33.3\% of cases [6]. The OS functions as the main drainage pathway when the lateral sinus is rudimentary [15].

Address for correspondence: Dr.Med., Dr.Biol., Dr.Hab. Professor of Anatomy, M.C. Rusu, Chair of the Division of Anatomy, Faculty of Dental Medicine, "Carol Davila" University of Medicine and Pharmacy, Bucharest, Romania, e-mail: anatomon@gmail.com; mugurel.rusu@umfcd.ro This article is available in open access under Creative Common Attribution-Non-Commercial-No Derivatives 4.0 International (CC BY-NC-ND 4.0) license, allowing to download articles and share them with others as long as they credit the authors and the publisher, but without permission to change them in any way or use them commercially. 

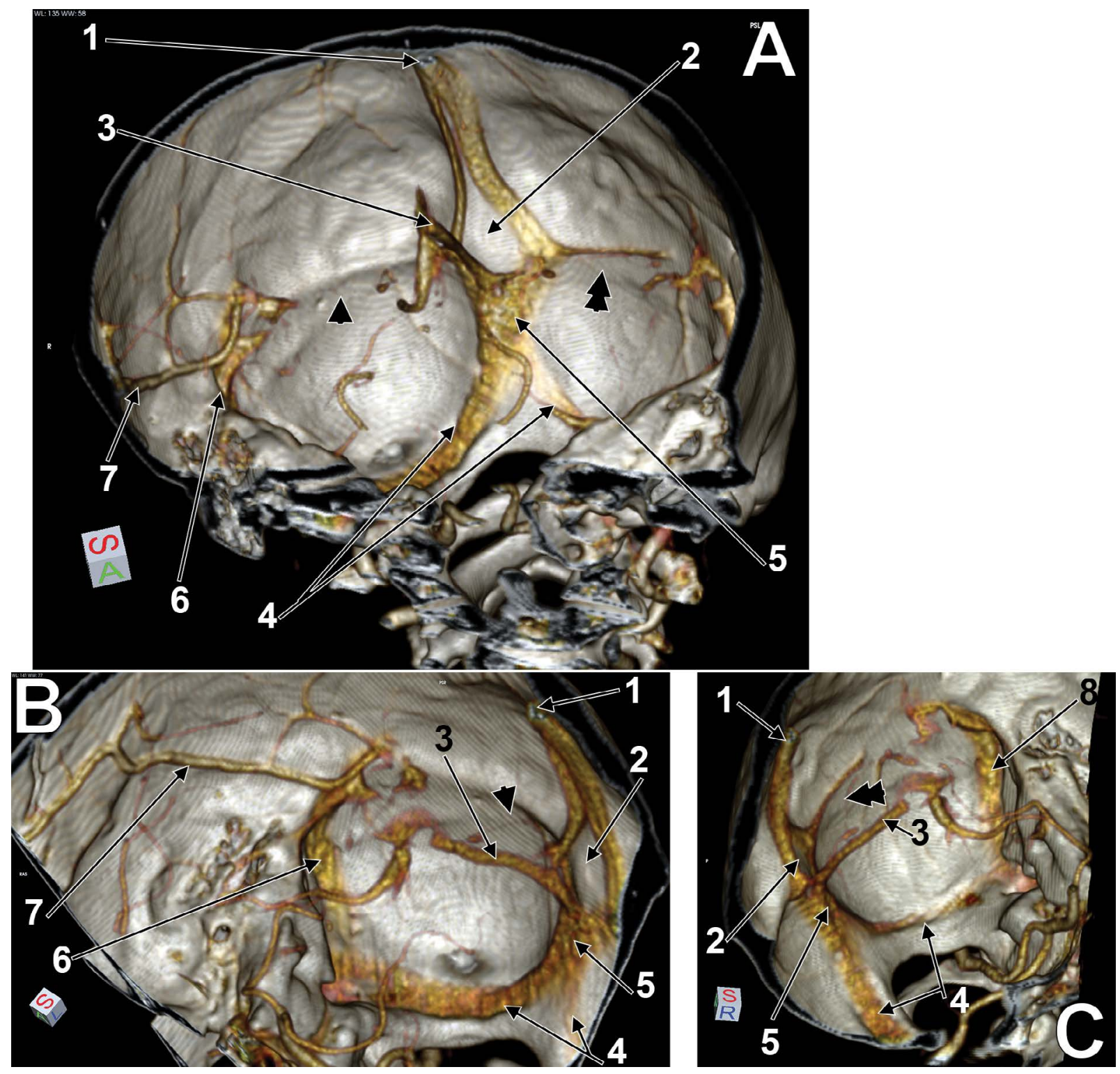

Figure 1. Three-dimensional volume renderings of the posterior fossa; A. Left antero-superior view; B. Left superior view; C. Right superior view; 1 - superior sagittal sinus; 2 - internal occipital protuberance; 3 - straight sinus; 4 - marginal sinuses; 5 - occipital sinus; 6 right sigmoid sinus; 7 - vein of Labbé; 8 - left sigmoid sinus. On the right side the groove for transverse sinus lacks and there is aplasia of that sinus (arrowhead). On the left side the groove for transverse sinus lacks and that sinus is hypoplastic (double arrowhead).

\section{CASE REPORT}

A 64-year-old female was admitted to the neurosurgical department of the National Institute of Neurology and Neurovascular Diseases for further diagnosis and treatment. For evaluation of possible vascular malformations a brain computed tomography angiography was performed after injecting iodine radiocontrast agent into the cubital fossa veins, followed by saline medium. The scan was performed with a 32-slice machine (Siemens Multislice Perspective Scanner), using a $0.6 \mathrm{~mm}$ collimation and reconstruction of $0.75 \mathrm{~mm}$ thickness with $50 \%$ overlap for multiplanar, maximum intensity projection (MIP) and three-dimensional volume rendering (3D-VR) technique, as described previously [12, 13]. The case was documented using the Horos for iOS software (Horos Project). The research was conducted ethically in accordance with The Code of Ethics of the World Medical Association (Declaration of Helsinki).

On the inner side of the cranial vault, the SSS was identified and was further tracked posteriorly. At about $4 \mathrm{~cm}$ above the internal occipital protuberance the SSS was dividing into a left arm $0.50 \mathrm{~cm}$ thick and a right arm $0.23 \mathrm{~cm}$ thick. These two arms di- 


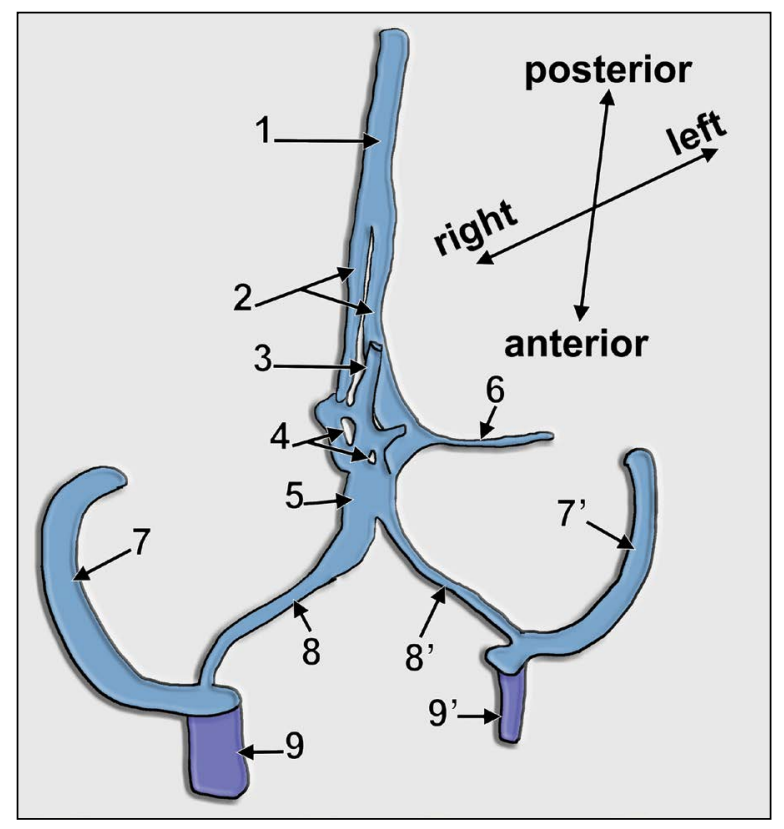

Figure 2. The confluent of the venous sinuses is drained via the occipito-marginal sinus. Right transverse sinus aplasia and left transverse sinus hypoplasia. Schema of the anatomic variant. Right anterior view; 1 - superior sagittal sinus; 2 - postero-inferior end of the superior sagittal sinus, divided into two divergent arms; 3 - straight sinus; 4 - venous network; 5 - occipital sinus; 6 - left hypoplastic transverse sinus, blind-ended; $7,7^{\prime}$ - sigmoid sinuses; 8, 8' - marginal sinuses; 9, 9' - internal jugular veins.

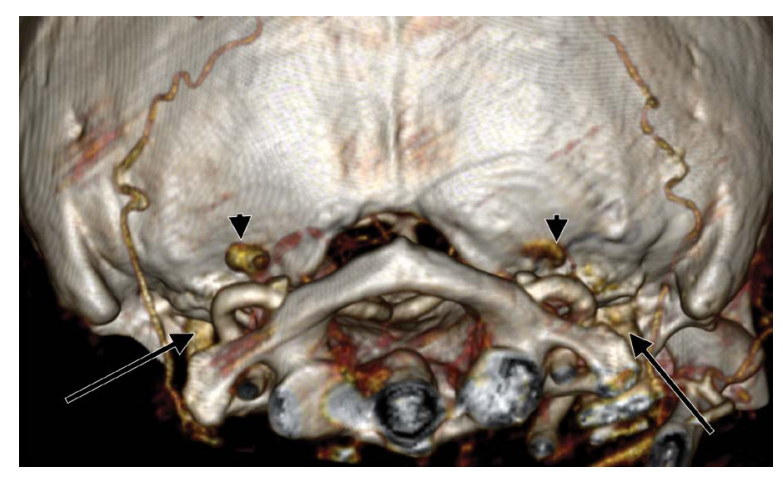

Figure 3. Three-dimensional volume rendering, postero-inferior view of the occipitovertebral junction. There are identified bilateral emissary condylar veins (arrowheads) and the internal jugular veins (arrows).

verged on the respective sides of the internal occipital protuberance, thus forming the superior borders of a venous rhomb (Figs. 1, 2). The right transverse sinus (TS) lacked (aplasia/agenesis), as also did the corresponding groove on the occipital squama. From the left angle of the venous rhomb left a hypoplastic $\mathrm{TS}$, the length of which was $2.28 \mathrm{~cm}$ and the calibre $0.11 \mathrm{~cm}$ calibre; it was blind-ended. The inferior borders of the venous rhomb joined inferior to the internal occipital protuberance to form the occipital sinus (OS) measuring $1.22 \mathrm{~cm}$. The straight sinus was connected with the right angle of the venous rhomb and with the right infero-lateral border of the venous rhomb through a venous network. The OS further divided on the sides of the vermian fossa into the left and right marginal sinuses (MSs). These MS on each side ended into the respective sigmoid sinus (SS) proximally to the jugular bulb (JB). From the SS-JB junction left, on each side, condylar emissary veins coursing through posterior condylar canals (Fig. 3).

\section{DISCUSSION AND CONCLUSIONS}

A study of the evolution of venous sinuses drainage in hominids brought arguments suggesting that selection for bipedalism determined epigenetic adaptations, such as a large OS-MS path and available emissary veins, but after bipedalism became established these adaptations relaxed [5]. It is interesting to consider also the ontogenesis of the OS. It is not been developed yet in 3-month-old foetuses [6]. A couple of months later, the TS is enlarged and reaches the torcular but the SiS is underdeveloped; drainage is ensured by OS channels connected with the MS and the jugular sinus [6]. The OS regresses only after birth [6] when a shift from a fatal to a postnatal type of circulation occurs [11]. Therefore, an OS-to-IJV anatomical path of drainage could be regarded as a persistent foetal morphology. Interestingly, a dissection study in 33 neonatal cadavers found no hypoplastic or aplastic sinuses, all the specimens had OS, and no bilateral drainage of the OSs via MSs was found [9]. When the OS drains into the SiS, and not into the jugular bulb, is termed "oblique OS" [15]. Such previously reported rare morphologies $[6,14]$, including the present case, are presented in Figure 4.

Seemingly, the OS-MS pathway, as well as condylar emissary veins, are of use to compensate dysplastic TSs. Bergman's Encyclopedia of Human Anatomic Variation [1] quotes the report of Hamnett et al. [7] who found the bilateral absence of the TS (hypoplasia with contralateral aplasia), the OS providing the only drainage pathway for the SSS and the StS to the MSs and the jugular bulbs. That variant mostly corresponds to ours, the difference being that in the case reported here the SiSs also drained through condylar veins.

Dora and Zileli [4] documented different combined variants, such as bilateral hypoplastic lateral sinuses, but with OS absent, or hypoplastic lateral 


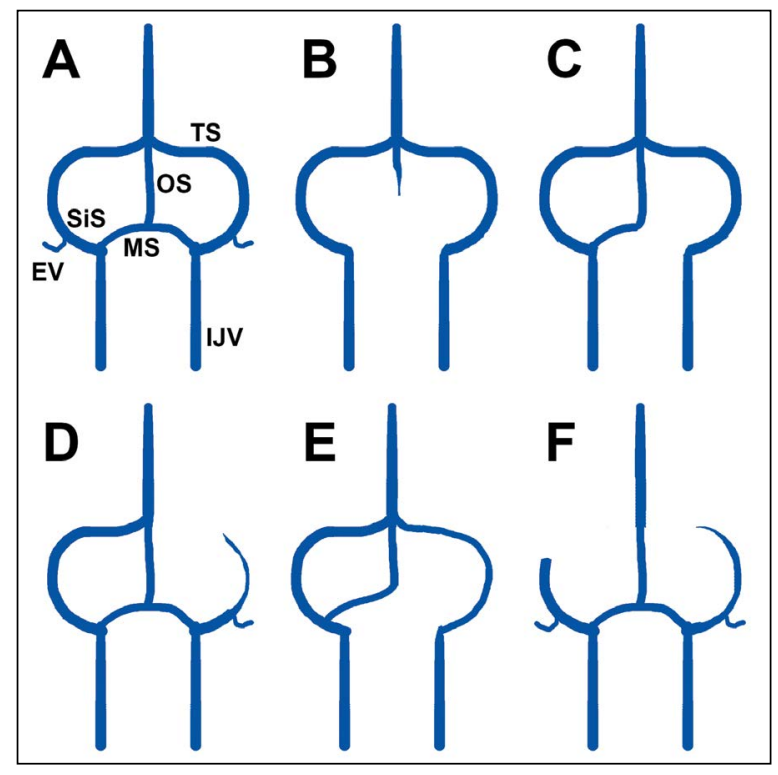

Figure 4. Different morphologies of the venous sinuses of the posterior fossa are compared to the complete prenatal one (A); B. Rudimentary OS, absent MSs; C. The OS drains unilaterally into the IJV bulb by a unilateral MS; $\mathbf{D}$. The OS drains bilaterally through MSs into the IJVs but one of the SiSs is hypoplastic/aplastic and that drainage is compensated through an EV; $E$. The OS drains unilaterally through a MS into the SiS, and not the jugular bulb (oblique OS); $\mathbf{F}$ (present case). The OS drains bilaterally into the IJVs, aplastic and hypoplastic TSs are compensated with bilateral EVs; TS — transverse sinus; SiS - sigmoid sinus; $\mathrm{OS}$ - occipital sinus; $\mathrm{MS}$ marginal sinus; EV — emissary vein; IJV — internal jugular vein.

sinus with OS present, but without MSs. None of the variants documented by these authors fits with the presently reported one.

A drainage pathway via the OS and MS with aplastic/hypoplastic TSs, such as in this case, stands as a unique possibility of emptying the confluent of sinuses. Therefore, surgical transection of the OS and/or MS would determine major perturbations in the venous drainage of the brain. The emissary veins could not compensate the resulted deficit because they are inserted into a variant of SiS unfilled from the confluent of sinuses.

Previously, in 1881, Knott [8] documented anatomical variants of the venous sinuses and discussed that both lateral sinuses, thus the TS continued as SiS, could be hypoplastic, the blood being drained via the OS and the MS. In the case reported here a TS was aplastic, and not hypoplastic.

The venous pattern of drainage is important in understanding the pathophysiology and risks associated with dural arteriovenous fistulas [10].

Conflict of interest: None declared

\section{REFERENCES}

1. Bergman RA, Tubbs RS, Shoja MM, Loukas M. Bergman's comprehensive encyclopedia of human anatomic variation. John Wiley \& Sons, Hoboken, New Jersey 2016.

2. Curé J, Van Tassel P, Smith M. Normal and variant anatomy of the dural venous sinuses. Seminars in Ultrasound, CT and MRI. 1994; 15(6): 499-519, doi: 10.1016/s08872171(05)80019-8.

3. Das S, Abd Latiff A, Suhaimi FH, et al. An anatomico-radiological study of the grooves for occipital sinus in the posterior cranial fossa. Bratisl Lek Listy. 2008; 109: 520-524, indexed in Pubmed: 19205565.

4. Dora F, Zileli T. Common variations of the lateral and occipital sinuses at the confluens sinuum. Neuroradiology. 1980; 20(1): 23-27, doi: 10.1007/BF00346857, indexed in Pubmed: 7422118.

5. Falk D. Evolution of cranial blood drainage in hominids: enlarged occipital/marginal sinuses and emissary foramina. Am J Phys Anthropol. 1986; 70(3): 311-324, doi: 10.1002/ajpa.1330700306, indexed in Pubmed: 3092672.

6. Fukusumi A, Okudera T, Takahashi S, et al. Anatomical evaluation of the dural sinuses in the region of the torcular herophili using three dimensional CT venography. Acad Radiol. 2010; 17(9): 1103-1111, doi: 10.1016/j. acra.2010.04.020, indexed in Pubmed: 20619699.

7. Hamnett NTJ, Ogungbo B, Nahser H, et al. Anomalous cerebral venous sinus drainage. $\mathrm{Br} J$ Neurosurg. 2010; 24(4): 497-498, doi: 10.3109/02688697.2010.489657, indexed in Pubmed: 20726761.

8. Knott JF. On the cerebral sinuses and their variations. J Anat Physiol. 1881; 16: 27-42, indexed in Pubmed: 17231415.

9. Kopuz C, Aydin ME, Kale A, et al. The termination of superior sagittal sinus and drainage patterns of the lateral, occipital at confluens sinuum in newborns: clinical and embryological implications. Surg Radiol Anat. 2010; 32(9): 827-833, doi: 10.1007/s00276-010-0628-2, indexed in Pubmed: 20182724.

10. McDougall CG, Halbach VV, Dowd CF, et al. Dural arteriovenous fistulas of the marginal sinus. 1997; 18: 1565-1572, indexed in Pubmed: 9296201.

11. Okudera T, Huang YP, Ohta T, et al. Development of posterior fossa dural sinuses, emissary veins, and jugular bulb: Morphological and radiologic study. Am J Neuroradiol. 1994; 15: 1871-1883, indexed in Pubmed: 7863937.

12. Rădoi PM, Rusu MC, Dincă D, et al. Combined rare anatomic variants: persistent primitive olfactory artery and azygos pericallosal artery. Surg Radiol Anat. 2021; 43(8): 1305-1308, doi: 10.1007/s00276-021-02687-9, indexed in Pubmed: 33496800.

13. Rusu MC, Măru N, Rădoi PM, et al. Trifurcated external carotid artery and complete gamma-loop of its maxillary branch. Surg Radiol Anat. 2019; 41(2): 231-234, doi: 10.1007/ s00276-018-2142-x, indexed in Pubmed: 30483866.

14. Tanoue S, Kiyosue $H$, Sagara $Y$, et al. Venous structures at the craniocervical junction: anatomical variations evaluated by multidetector row CT. Br J Radiol. 2010; 83(994): 831-840, doi: 10.1259/bjr/85248833, indexed in Pubmed: 20647517.

15. Tubbs RS, Bosmia AN, Shoja MM, et al. The oblique occipital sinus: a review of anatomy and imaging characteristics. Surg Radiol Anat. 2011; 33(9): 747-749, doi: 10.1007/ s00276-011-0831-9, indexed in Pubmed: 21626273. 